\title{
Online Appendix \\ Bayesian Model Averaging for Dynamic Panels with an Application to a Trade Gravity Model
}

\author{
Huigang Chen* ${ }^{*} \quad$ Alin Mirestean ${ }^{\dagger} \quad$ Charalambos G. Tsangarides ${ }^{\ddagger}$
}

February 24, 2016

\footnotetext{
*MarketShare Partners; Email: hchen@marketshare.com

†International Monetary Fund; Email: amirestean@imf.org.

${ }^{\ddagger}$ Corresponding author. International Monetary Fund, Research Department, HQ1-9-612B, 700 19th Street, N.W., Washington D.C. 20431, U.S.A.; Email: ctsangarides@imf.org.
} 


\section{Appendix A: Robustness using non-Gaussian errors}

In this Appendix we relax the normality assumption for the idiosyncratic random error, $v_{i t}$, and perform another Monte Carlo experiment to evaluate the model averaging and model selection properties. Results are discussed in Section 4 of the paper.

Table A1. Posterior probability of the true model LIBMA summary statistics for various $N, \alpha$, and $\sigma_{v}^{2}$

\begin{tabular}{|c|c|c|c|c|c|c|c|}
\hline \multirow[b]{3}{*}{ Sample } & \multirow{3}{*}{$\begin{array}{r}\alpha \\
\sigma_{v}^{2}\end{array}$} & \multicolumn{3}{|c|}{0.95} & \multicolumn{3}{|c|}{0.50} \\
\hline & & 0.05 & 0.10 & 0.20 & 0.05 & 0.10 & 0.20 \\
\hline & & & & & & & \\
\hline \multicolumn{8}{|l|}{$N=200$} \\
\hline Mean & & 0.224 & 0.109 & 0.044 & 0.162 & 0.084 & 0.031 \\
\hline Variance & & 0.021 & 0.012 & 0.004 & 0.025 & 0.015 & 0.005 \\
\hline Q1 & & 0.094 & 0.025 & 0.008 & 0.019 & 0.002 & 0.000 \\
\hline Median & & 0.222 & 0.071 & 0.023 & 0.115 & 0.023 & 0.003 \\
\hline Q3 & & 0.342 & 0.161 & 0.053 & 0.283 & 0.118 & 0.023 \\
\hline \multicolumn{8}{|l|}{$N=500$} \\
\hline Mean & & 0.459 & 0.415 & 0.253 & 0.437 & 0.376 & 0.243 \\
\hline Variance & & 0.023 & 0.025 & 0.028 & 0.032 & 0.036 & 0.035 \\
\hline Q1 & & 0.371 & 0.315 & 0.110 & 0.322 & 0.233 & 0.069 \\
\hline Median & & 0.497 & 0.447 & 0.231 & 0.487 & 0.412 & 0.211 \\
\hline Q3 & & 0.580 & 0.543 & 0.386 & 0.582 & 0.534 & 0.397 \\
\hline \multicolumn{8}{|l|}{$N=2000$} \\
\hline Mean & & 0.653 & 0.632 & 0.643 & 0.640 & 0.643 & 0.651 \\
\hline Variance & & 0.021 & 0.025 & 0.025 & 0.026 & 0.025 & 0.024 \\
\hline Q1 & & 0.607 & 0.573 & 0.585 & 0.592 & 0.595 & 0.598 \\
\hline Median & & 0.703 & 0.684 & 0.703 & 0.700 & 0.699 & 0.708 \\
\hline Q3 & & 0.754 & 0.747 & 0.751 & 0.753 & 0.754 & 0.757 \\
\hline
\end{tabular}

Notes:

1. The table presents means, variances, Q1, median (Q2), and Q3 of the posterior probability of the data generating process (true model) across the Monte Carlo replications. Higher values indicate that the true model is favored. We consider sample sizes $N=200,500,2000$, two values for the coefficient of the lagged dependent variable, $a=0.95,0.50$, and three values for the variance of the idiosyncratic random error, $\sigma_{v}^{2}=0.05,0.10,0.20$.

2. The error terms are constructed using discrete distributions (see Section 4.1.). 
Table A2. Posterior probability ratio of true model versus best among the rest LIBMA summary statistics for various $N, \alpha$, and $\sigma_{v}^{2}$

\begin{tabular}{|c|c|c|c|c|c|c|c|}
\hline \multirow{3}{*}{ Sample } & \multirow{3}{*}{$\begin{array}{r}\alpha \\
\sigma_{v}^{2}\end{array}$} & \multicolumn{3}{|c|}{0.95} & \multicolumn{3}{|c|}{0.50} \\
\hline & & 0.05 & 0.10 & 0.20 & 0.05 & 0.10 & 0.20 \\
\hline & & & & & & & \\
\hline \multicolumn{8}{|l|}{$N=200$} \\
\hline Mean & & 1.587 & 0.828 & 0.350 & 1.205 & 0.662 & 0.254 \\
\hline Variance & & 1.767 & 1.043 & 0.348 & 1.813 & 1.168 & 0.420 \\
\hline Q1 & & 0.463 & 0.168 & 0.053 & 0.099 & 0.011 & 0.001 \\
\hline Median & & 1.235 & 0.423 & 0.173 & 0.639 & 0.141 & 0.016 \\
\hline Q3 & & 2.499 & 1.081 & 0.357 & 1.975 & 0.812 & 0.162 \\
\hline \multicolumn{8}{|l|}{$N=500$} \\
\hline Mean & & 3.384 & 3.050 & 1.783 & 3.258 & 2.721 & 1.715 \\
\hline Variance & & 4.835 & 4.355 & 3.257 & 5.489 & 4.810 & 3.590 \\
\hline Q1 & & 1.532 & 1.318 & 0.437 & 1.205 & 0.867 & 0.258 \\
\hline Median & & 3.065 & 2.705 & 1.118 & 2.862 & 2.216 & 1.044 \\
\hline Q3 & & 5.035 & 4.584 & 2.630 & 5.069 & 4.278 & 2.487 \\
\hline \multicolumn{8}{|l|}{$N=2000$} \\
\hline Mean & & 6.980 & 6.410 & 6.992 & 6.828 & 6.824 & 7.081 \\
\hline Variance & & 18.234 & 17.732 & 19.169 & 19.496 & 18.475 & 19.192 \\
\hline Q1 & & 3.360 & 2.956 & 3.035 & 3.138 & 3.257 & 3.221 \\
\hline Median & & 6.676 & 5.738 & 6.824 & 6.335 & 6.464 & 6.762 \\
\hline Q3 & & 10.195 & 9.520 & 10.229 & 9.999 & 10.021 & 10.578 \\
\hline
\end{tabular}

Notes:

1. The table presents means, variances, Q1, median (Q2), and Q3 of the of the ratio of the posterior model probability of the true model to the highest posterior probability of all the other models (excluding the true model) across the Monte Carlo replications. Higher values indicate that the true model is favored. We consider sample sizes $N=200,500,2000$, two values for the coefficient of the lagged dependent variable, $a=0.95,0.50$, and three values for the variance of the idiosyncratic random error, $\sigma_{v}^{2}=0.05,0.10,0.20$.

2. The error terms are constructed using discrete distributions (see Section 4.1.). 
Table A3. Probability of retrieving the true model LIBMA summary statistics for various $N, \alpha$, and $\sigma_{v}^{2}$

\begin{tabular}{|c|c|c|c|c|c|c|c|}
\hline \multirow[b]{3}{*}{ Sample } & \multirow{2}{*}{$\begin{array}{r}\alpha \\
\sigma_{v}^{2}\end{array}$} & \multicolumn{3}{|c|}{0.95} & \multicolumn{3}{|c|}{0.50} \\
\hline & & 0.05 & 0.10 & 0.20 & 0.05 & 0.10 & 0.20 \\
\hline & & & & & & & \\
\hline$N=200$ & & & & & & & \\
\hline$\%$ Correct & & 56 & 27 & 8 & 42 & 22 & 7 \\
\hline$N=500$ & & & & & & & \\
\hline$\%$ Correct & & 85 & 80 & 53 & 79 & 72 & 51 \\
\hline$N=2000$ & & & & & & & \\
\hline$\%$ Correct & & 93 & 92 & 93 & 92 & 92 & 93 \\
\hline
\end{tabular}

\section{Notes:}

1. The table presents the percentage of the Monte Carlo replications that the true model has the highest posterior probability. Higher percentages suggest that the methodology recovers the true model more often. We consider sample sizes, $N=200,500$, 2000 , two values for the coefficient of the lagged dependent variable, $a=0.95,0.50$, and three values for the variance of the idiosyncratic random error, $\sigma_{v}^{2}=0.05,0.10,0.20$.

2. The error terms are constructed using discrete distributions (see Section 4.1.). 


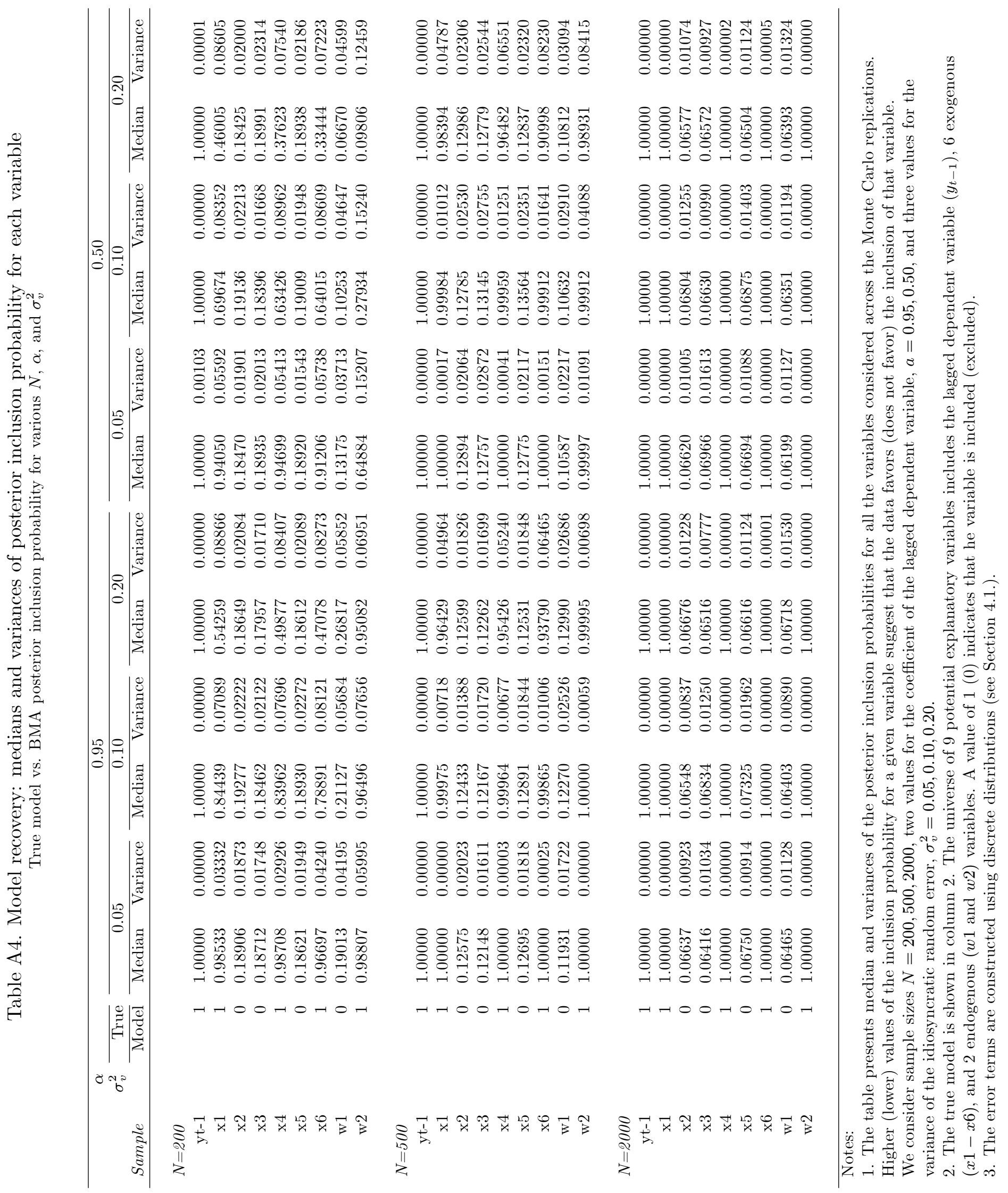



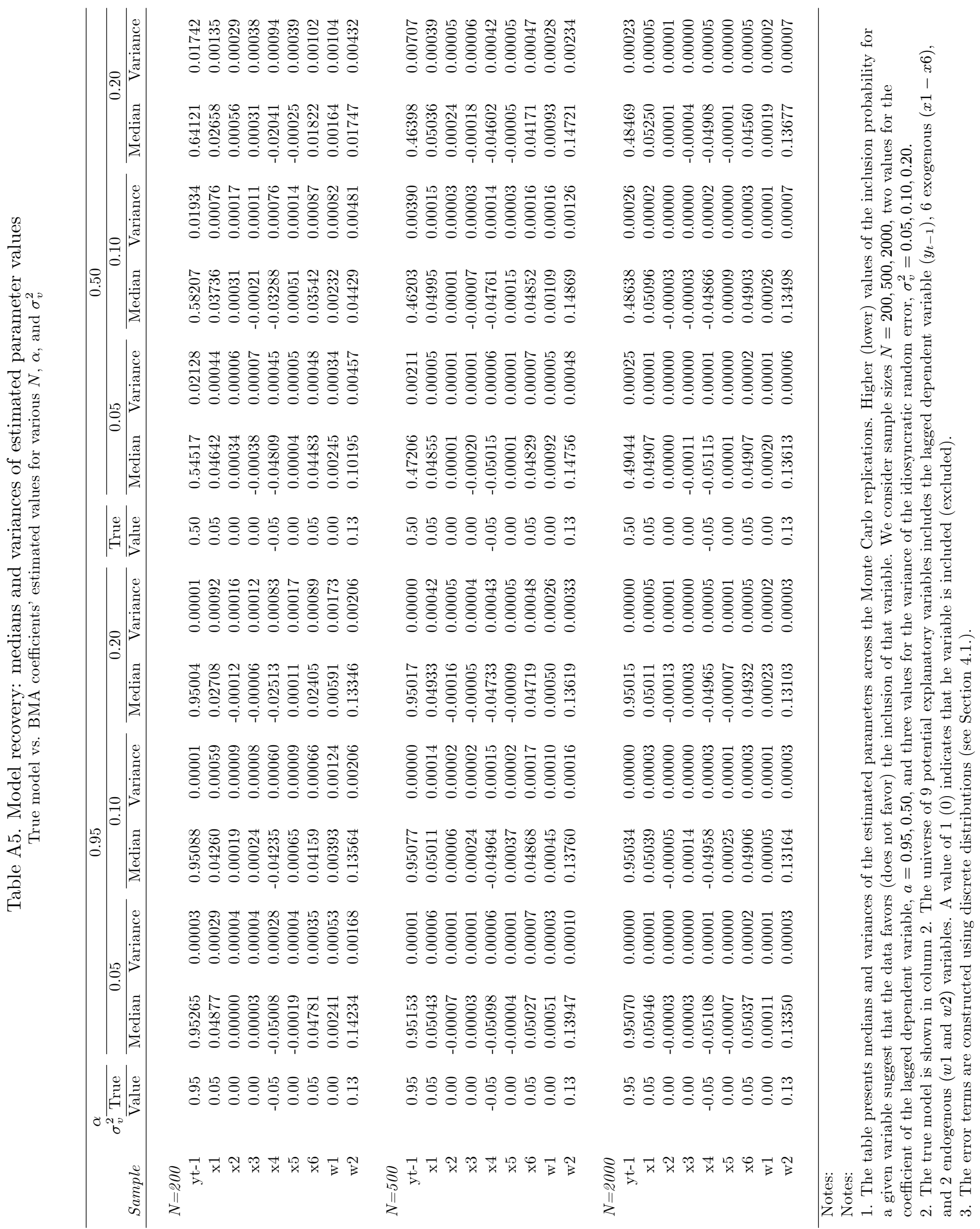


\section{Appendix B: LIBMA predictive properties}

In this Appendix, we examine the predictive properties of our methodology against more parsimonious models. Results are discussed in Section 4 of the paper. 
8

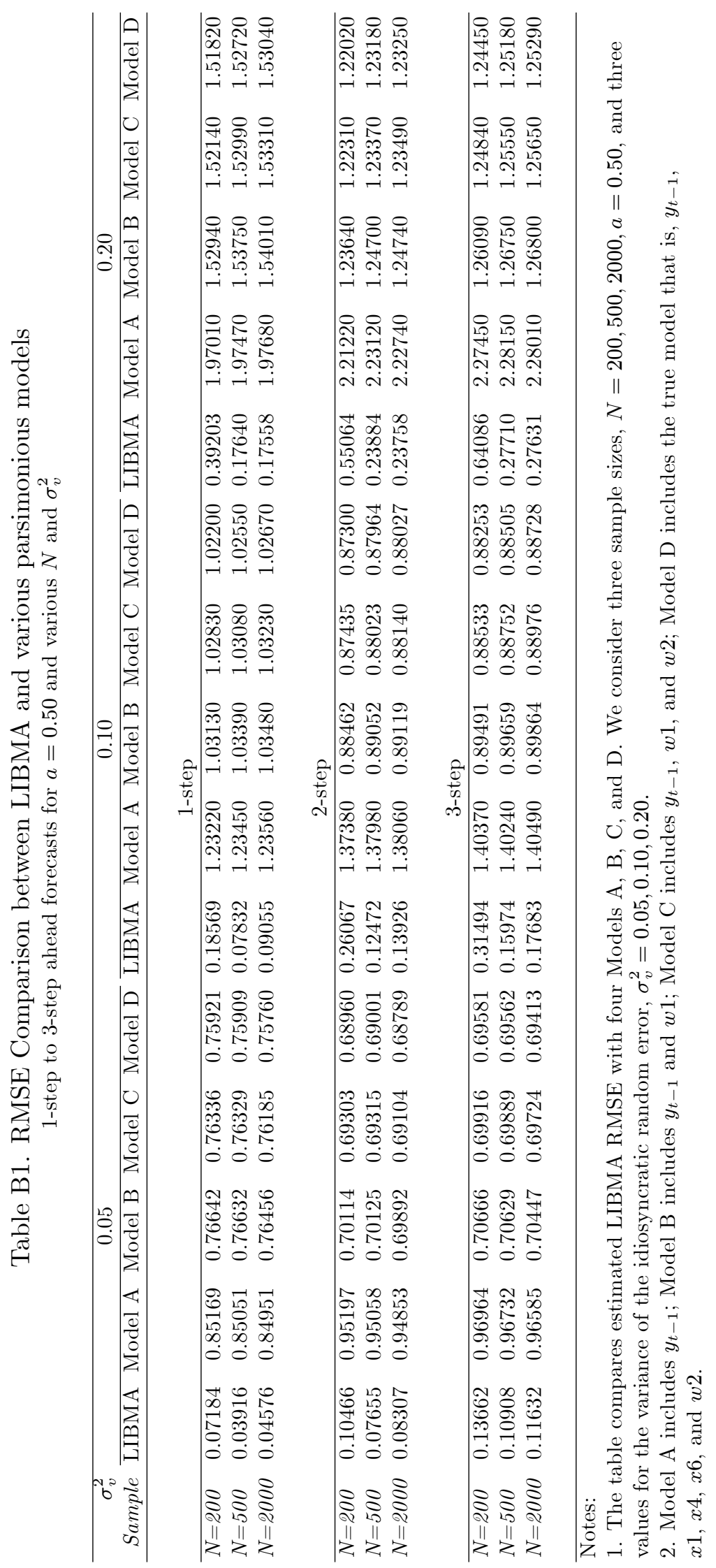

